\title{
Study on patients without underlying chronic liver disease to improve survival outcome in pediatric liver transplantation
}

\author{
Suk Kyun Hong, Nam-Joon Yi, Kwangpyo Hong, Eui Soo Han, Jeong-Moo Lee, YoungRok Choi, \\ Kwang-Woong Lee, Kyung-Suk Suh
}

Department of Surgery, Seoul National University Hospital, Seoul, Korea

Background: Although liver transplantation (LT) is currently the standard treatment for pediatric end-stage liver disease (PELD), it remains associated with significant complications. This study aimed to identify risk factors affecting the outcomes of pediatric LT.

Methods: Data from pediatric patients who underwent primary LT from March 1988 to December 2018 were retrospectively analyzed. Chronic liver disease was defined as an explanted liver showing fibrosis regardless of grade, cirrhosis, or any other underlying disease that may cause progressive liver injury leading to fibrosis or cirrhosis.

Results: A total of 255 pediatric patients underwent LT during the study period. Their 1-, 5-, and 10-year overall survival rates were $90.5 \%, 88.4 \%$, and $87.8 \%$, respectively, while the $1-, 5$-, and 10 -year graft survival rates were $89.7 \%, 88.5 \%$, and $87.2 \%$, respectively. Multivariate analysis showed that liver disease without underlying chronic liver disease $(P=0.024)$ and $P E L D$ score $\geq 30(P=0.036)$ were associated with worse survival. Furthermore, bodyweight $<6 \mathrm{~kg}(P=0.050)$, whole-liver deceased donor $L T$ compared to living donor $L T(P=0.008)$, fulminant liver failure $(P=0.008)$, and postoperative hepatic artery complications $(P<0.001)$ were risk factors for graft survival. Liver disease without underlying chronic liver disease was the only factor independently associated with hepatic artery complications $(P=0.003)$.

Conclusions: Greater caution is recommended in pediatric patients with liver disease unaccompanied by underlying chronic liver disease, high PELD score, or low body weight to improve survival after LT. Hepatic artery complications were the only surgical complications affecting graft survival, especially in patients with liver disease but no underlying chronic liver disease.

Corresponding author: Nam-Joon Yi

E-mail: gsleenj@hanmail.net

(c) The Korean Society for Transplantation

This is an Open Access article distributed under the terms of the Creative Commons Attribution Non-Commercial License (http://creativecommons.org/licenses/by-nc/4.0/) which permits unrestricted non-commercial use, distribution, and reproduction in any medium, provided the original work is properly cited. 